\title{
Teste da caminhada de seis minutos em pacientes com DPOC durante programa de reabilitação*
}

\author{
Maria Auxiliadora Carmo Moreira ${ }^{1}$, Maria Rosedália de Moraes², Rogério Tannus 3
}

Objetivos: Relatar resultados obtidos em testes da caminhada de seis minutos (TC6) de pacientes com DPOC integrantes de programa de reabilitação pulmonar (PRP), com objetivo de contribuir para interpretação e adequada utilização deste teste. Paralelamente, estudar alguns fatores que interferem no treinamento físico durante a reabilitação pulmonar. Materiais e métodos: Foram avaliados, retrospectivamente, dados de 18 homens e cinco mulheres integrantes do PRP. Os pacientes foram submetidos a treinamento dos membros inferiores por três meses em três sessões por semana. Alguns parâmetros pré e pós-treinamento foram analisados: distância percorrida no TC6 e sua relação com os valores calculados pelas equações de Enright e Sherril para valores de referência, volume expiratório forçado no $1^{0}$ segundo $\left(\mathrm{VEF}_{1}\right)$, carga de treinamento, freqüência cardíaca (FC) freqüência respiratória (f), saturação do oxigênio por oximetria $\left(\mathrm{SpO}_{2}\right)$, índice de massa corpórea (IMC). Resultados: Os valores obtidos durante 0 TC6 pré-treinamento nos pacientes não diferiram dos de referência resultantes das equações de Enright e Sherril $(p=0,777)$. Houve diferença significativa nesses valores, após treinamento $(p=0,001)$. 0 aumento médio, na distância percorrida no Tc6, após treinamento, foi de

$76 \mathrm{~m}$; contudo, três pacientes não atingiram o aumento mínimo significativo de $54 \mathrm{~m}$.

A distância percorrida, pós-treinamento correlacionou-se positivamente com $0 \mathrm{VEF}_{1}$ pré-treinamento $(p=0,012)$, mas não com $o$ incremento de carga durante $o$ treinamento $(p=0,693)$. Não se observou diferença significativa na $\mathrm{f}, \mathrm{SpO}_{2}$ no TC6 antes e após treinamento. A FC aumentou significantemente no final do TC6 após treinamento. Nove pacientes (39\%) apresentaram o IMC abaixo do limite inferior; destes, 6 (66\%) atingiram $54 \mathrm{~m}$ ou mais de incremento na distância caminhada após treinamento. Conclusão: Sugere-se utilizar-se metodologia padronizada e adequada para realização do TC6, inclusive quando o objetivo for obter valores de referência. As equações de Enright e Sherril para indivíduos normais parecem tender a valores subestimados, não diferindo estatisticamente dos valores obtidos pré-treinamento nesses pacientes com DPOC. Isso se deve, provavelmente, a diferenças na técnica de encorajamento utilizada pelos autores. Confirma-se o ganho de performance física, após reabilitação, independente do estado funcional inicial, justificando sua indicação mesmo para pacientes com limitação respiratória acentuada. A resposta heterogênea ao incremento de carga

sugere a necessidade de analisar fatores preditivos da melhor resposta ao treinamento, para personalizá-lo e obter a melhor performance possível dos pacientes. (J Pneumol 2001;27(6):295-300)

\section{Six minute walk test in COPD patients under a rehabilitation program}

Objectives: To report the results obtained in six-minute walk tests (6 MWT) performed in a group of patients under a pulmonary rehabilitation program (PRP) as a contribution to a better interpretation and the proper use of this test. Some factors that interfere with physical training during pulmonary rehabilitation were also studied. Methods: Retrospective data on 18 males and 5 females under a PRP were evaluated. During 3 months, the patients had 3 weekly training sessions to exercise the muscles of the lower extremities. The following parameters were analyzed before and after training: the distance walked during the 6 MWT and its relationship with the

* Trabalho realizado no Laboratório de Função Pulmonar e no Centro de Reabilitação e Fisioterapia Respiratória de Serviço de Pneumologia da Faculdade de Medicina da Universidade Federal de Goiás, Goiania, GO.

1. Professora Adjunta; Chefe do Serviço de Pneumologia.

2. Médica do Serviço de Pneumologia.
3. Fisioterapeuta do Hospital das Clínicas.

Endereço para correspondência - Serviço de Pneumologia - Hospital das Clínicas - UFG, 1a Av. S/N, Setor Universitário - 74370-210 Goiânia, GO. E-mail: helpuol@uol.com.br

Recebido para publicação em 15/6/00. Aprovado, após revisão, em 18/6/01. 
reference values calculated by the equations of Enright and Sherril; forced expiratory volume in one second, training load, heart rate (HR), respiratory rate (RR), oxygen saturation by pulse oxymeter $\left(\mathrm{SpO}_{2}\right)$ and body mass index (BMI). Results: The values obtained during pre-training 6 MWT did not differ from the reference values calculated by Enright and Sherril equations $(P=$ 0.777). After training, these values differed significantly $(P=0.001)$. The mean 6 MWT distance increase was $76 \mathrm{~m}$. However, 3 patients did not reach the minimally significant increase of $54 \mathrm{~m}$. Post-training 6 MWT correlated positively with pre-training FEV ${ }_{1}(P=0.012)$, but not with load increase during training $(P=0.693)$. No significant difference in $\mathrm{RR}$ and $\mathrm{SpO}_{2}$ was observed before and after training. HR increased significantly at the end of post-training 6 MWT $(P=0.035)$.

In 9 patients BMI was lower than the inferior limit. Six of them $(66 \%)$ presented post-training distance increased in $54 \mathrm{~m}$ or more. Conclusions: The use of standardized methods for 6 MWT performance is recommended, even when reference values are targeted. The equations of Enright and Sherril for healthy subjects tended to yield underestimated values, with no statistical differences in training values in COPD patients. Differences in the encouragement technique employed could explain these conflicting results. Physical gain after rehabilitation was confirmed regardless of the patient's initial functional status, justifying the indication of rehabilitation even to those with severe respiratory limitations. The heterogeneous responses to load increase suggest the analysis of the predictive factors of the best response to training is necessary to customize load increase and thus obtain the best performance possible.

Descritores - Teste de esforço. Pneumopatias obstrutivas. Reabilitação. Testes de função respiratória. Exercícios.

Key words - Exercise test. Obstructive lung diseases. Rehabilitation. Respiratory function tests. Exercise.

\section{INTRODUÇÃO}

0 conceito de reabilitação remonta à década de 40 , quando foi considerada abordagem que pressupõe a visão ampla do indivíduo (clínica, emocional, social e vocacional). Todavia, somente na década de 70 os programas de reabilitação pulmonar (PRP) ganharam maior aceitação e se tornaram tema de inúmeros estudos.

À medida que um maior número de programas foi criado, surgiu a preocupação de padronizar e melhor estudar as técnicas e métodos nos protocolos utilizados(1).

0 teste da caminhada de seis minutos (TC6) tem sido preconizado e utilizado na avaliação de resultados de programa de reabilitação. É um teste simples e facilmente realizado. Todavia, são ainda escassos os relatos e discussões quanto à padronização da técnica e fatores que interferem na sua efetividade. Equações para cálculo de valores de referência para distância caminhada recentemente começaram a ser publicadas. U tilizamos neste trabalho as equações de referência de Enright e Sherril(2). Contudo, observamos diferenças na técnica de encorajamento dos pacientes relatada pelos autores em relação ao nosso protocolo.

Vários parâmetros, normalmente avaliados durante PRP, devem ser melhor discutidos devido ao potencial para influenciarem no resultado do treinamento e no TC6. Dentre eles: volume expiratório forçado no primeiro segun-
Siglas e abreviaturas utilizadas neste trabalho

DPOC - Doença pulmonar obstrutiva crônica

$f$ - Freqüência respiratória

FC - Freqüência cardíaca

IMC - Índice da massa corpórea $\left(\mathrm{kg} / \mathrm{m}^{2}\right)$

MMII - Membros inferiores

MMSS - Membros superiores

PRP - Programa de reabilitação pulmonar

$\mathrm{SpO}_{2}$ - Saturação arterial de oxigênio por oximetria de pulso

TC6 - Teste da caminhada de seis minutos

$V E F_{1}$ - Volume expiratório forçado no $1^{\text {o }}$ segundo da capacidade vital

do, carga de treinamento, $\mathrm{FC}, \mathrm{f}, \mathrm{SpO}_{2}$ e parâmetros nutricionais.

Parece plausível que a publicação em maior escala de resultados de programas de reabilitação poderá contribuir para a padronização e melhor avaliação dos métodos empregados.

O objetivo deste estudo é contribuir para somar subsídios à padronização, interpretação e utilização do Tc6, e, paralelamente, discutir, utilizando os parâmetros disponíveis, alguns fatores que interferem no treinamento físico durante 0 PRP.

\section{Material e mÉTOdos}

Estudamos, retrospectivamente, dados dos prontuários de 23 pacientes com DPOC, 18 do sexo masculino e cinco de feminino, que participaram do PRP em nosso serviço.

Foram escolhidos, dentro dos parâmetros disponíveis, aqueles cujas alterações ou magnitude pudessem interferir no resultado do TC6. Foram estudados o volume expi- 
ratório forçado no primeiro segundo da capacidade vital $\left(\mathrm{VEF}_{1}\right)$, incremento de carga na esteira no final do treinamento, freqüência cardíaca $(F C)$, freqüência respiratória (f), saturação arterial da oxigênio por oxímetro de pulso $\left(\mathrm{SpO}_{2}\right)$ e índice da massa corpórea (IMC). Todos os parâmetros anteriores, com exceção do IMC e $V_{E F}$, se referem aos períodos pré e pós-treinamento no início e no final do TC6. 0 IMC foi obtido antes e $0 \mathrm{VEF}_{\mathrm{i}}$ antes e após treinamento.

O programa de reabilitação incluiu: avaliação inicial pneumológica, nutricional, fisioterápica e psicológica. Todos realizaram teste ergométrico e espirometria. 0 período de treinamento compreendeu 12 semanas com sessões três vezes por semana. Os pacientes receberam 0 treinamento de membros inferiores (MMII), em esteira com velocidade $60 \%$ daquela atingida no teste incremental, que foi realizado mensalmente, em esteira ergométrica e limitado por sintomas utilizando-se a escala de Borg. Foi realizado teste incremental de membros superiores (MMSS) limitado por sintomas, com uso de halteres, seguido de treinamento de mMSS por período igual àquele de MMII. Em todas as sessões foram realizados exercícios intervalados, de alongamento e uma vez por semana de relaxamento.

A espirometria foi realizada utilizando-se 0 equipamento Vitatrace-VT 130 segundo critérios da American Thoracic Society (ATS) e Consenso Brasileiro de Espirometria ${ }^{(3,4)}$.

Utilizou-se para o teste incremental uma esteira Accusmart Weslo Caden 840 , com inclinação fixa de $6 \%$, velocidade inicial de uma milha por hora, com incrementos de meia milha a cada dois minutos até o máximo tolerado, baseando-se na escala de Borg. Monitorizou-se pressão arterial (PA), freqüência respiratória (f) e saturação de oxigênio por oximetria de pulso $\left(\mathrm{SpO}_{2}\right)$.

$O$ teste da caminhada de seis minutos foi realizado com monitorização da pressão arterial, $\mathrm{FC}$, f e $\mathrm{SpO}_{2}$, em um corredor plano, com distâncias previamente demarcadas. 0 paciente foi acompanhado por um membro da equipe de reabilitação e incentivado, por estímulo verbal constante, a andar o mais rápido possível. Q uando o paciente interrompia a caminhada, o cronômetro continuava acionado.

A medida da $\mathrm{SpO}_{2}$ foi realizada com oxímetro marca Dixtal modelo Superbright-DX 2455, com sensor posicionado no 3 o dedo da mão direita, sendo a leitura determinada após estabilização do sinal. No mesmo momento obtinha-se a freqüência cardíaca (FC).

Foram coletados dados referentes a idade, sexo, altura e peso, velocidade de treinamento no início e final do programa, distância percorrida no teste da caminhada antes e após treinamento.
U tilizaram-se para cálculo do valor previsto, ou de referência, para distância no TC6, as equações propostas por Enright e Sherril(2), determinando-se o percentual do previsto para cada teste realizado pelo paciente:

H omens: distância Tc $6(\mathrm{~m})=(7,57 \times$ altura $\mathrm{cm})-(5,02$ $x$ idade $)-(1,76 x$ peso $\mathrm{kg})-309 \mathrm{~m}$.

Mulheres: distância Tc6 $(\mathrm{m})=(2,11 \times$ altura $\mathrm{cm})-(2,29$ $x$ peso $\mathrm{kg})-(5,78 \mathrm{x}$ idade $)+667 \mathrm{~m}$.

0 IMC (índice de Quetelet) foi calculado através da fórmula clássica: $I M C=$ peso atual $(\mathrm{kg}) / \mathrm{A}^{2}(\mathrm{~m})$. Consideraram-se os seguintes limites inferiores de normalidade: IMC $<21$ para faixa etária de 35 a 44 anos; < 22 para 45 a 54 anos; < que 23 para 55 a 64 anos e < 24 para 65 anos ou mais ${ }^{(5)}$.

Considerou-se melhora do teste da caminhada um incremento de $54 \mathrm{~m}$ em relação ao teste pré-treinamento(6).

Análise estatística: Para análise estatística foram utilizados: 1) teste t de Student; 2) análise de variância; 3) coeficiente de correlação de Pearson. $\mathrm{Na}$ analise de correlação utilizou-se o teste $\mathrm{t}$.

\section{Resultados}

Os dados antropométricos e demográficos estão listados na Tabela 1.

Cinco pacientes não concluíram o programa de reabilitação; portanto, seus dados foram considerados somente para análise da relação $\operatorname{VEF}_{1}$ pré-treinamento e a distância percorrida pós-treinamento.

Os resultados relacionados ao período de treinamento e após treinamento estão listados na Tabela 2.

Observou-se aumento médio após treinamento em $76 \mathrm{~m}$ na distância caminhada no Tc6. Apenas três pacientes (16\%) não atingiram o patamar de $54 \mathrm{~m}$, considerado aumento significativo neste teste (Figura 1 ).

$\mathrm{Não}$ foi detectada diferença significante entre os valores da distância caminhada, obtidos no teste da caminhada pré-treinamento e os valores previstos obtidos pela equação de Enright e Sherril ( $t=0,287 ; p=0,777)$. Os valores pós-treinamento diferiram significantemente dos previstos ( $t=3,830 ; p=0,001$ ) (Figura 2 ).

Os valores do $\mathrm{VEF}_{1}$ pré e pós-treinamento não diferiram significantemente $(t=1,057 ; p=0,307)$. Não se obteve correlação entre $\mathrm{VEF}_{1}$ e distância percorrida prétreinamento $(t=0,516 ; p=0,611)$. Contudo, a distância

\section{TABELA 1}

Parâmetros antropométricos e demográficos do grupo estudado

\begin{tabular}{lrc}
\hline Parâmetro & Média & Desvio padrão \\
\hline Idade $(\mathrm{a})$ & 65,0 & 7,6 \\
Peso $(\mathrm{kg})$ & 61,9 & 12,3 \\
Altura $(\mathrm{cm})$ & 161,2 & 8,3 \\
IMC & 23,8 & 4,1 \\
\hline
\end{tabular}


TABELA 2

Parâmetros relacionados aos períodos pré e pós-treinamento

\begin{tabular}{|c|c|c|c|c|c|c|c|}
\hline \multirow[t]{2}{*}{ Parâmetro } & \multicolumn{3}{|c|}{ Pré } & \multicolumn{3}{|c|}{ Pós } & \multirow[t]{2}{*}{$\mathbf{p}$} \\
\hline & $\mathbf{N}$ & Média & DP & $\mathbf{N}$ & Média & DP & \\
\hline $\begin{array}{l}\text { Dist. percorrida } \\
\text { (m e \% do previsto) }\end{array}$ & 23 & $499,0(99)$ & 88,5 & 18 & $575,0(114)$ & 67,5 & $<0,05$ \\
\hline $\operatorname{VEF}_{1}$ (\% do previsto) & 23 & 45,8 & 16,0 & 18 & 49,0 & 17,5 & NS \\
\hline Veloc. esteira km/h & 18 & 3,2 & 1,0 & 18 & 5,1 & 2,1 & $<0,05$ \\
\hline $\mathrm{FC}$ & 18 & 107,1 & 17,2 & 18 & 116,5 & 10,5 & $<0,05$ \\
\hline$f$ & 18 & 27,6 & 5,4 & 18 & 28,0 & 5,3 & NS \\
\hline $\mathrm{SpO}_{2}$ & 18 & 89,3 & 5,2 & 18 & 88,4 & 6,1 & NS \\
\hline
\end{tabular}

- FC, f, $\mathrm{SpO}_{2}$ pós-TC6, pré e pós-treinamento.

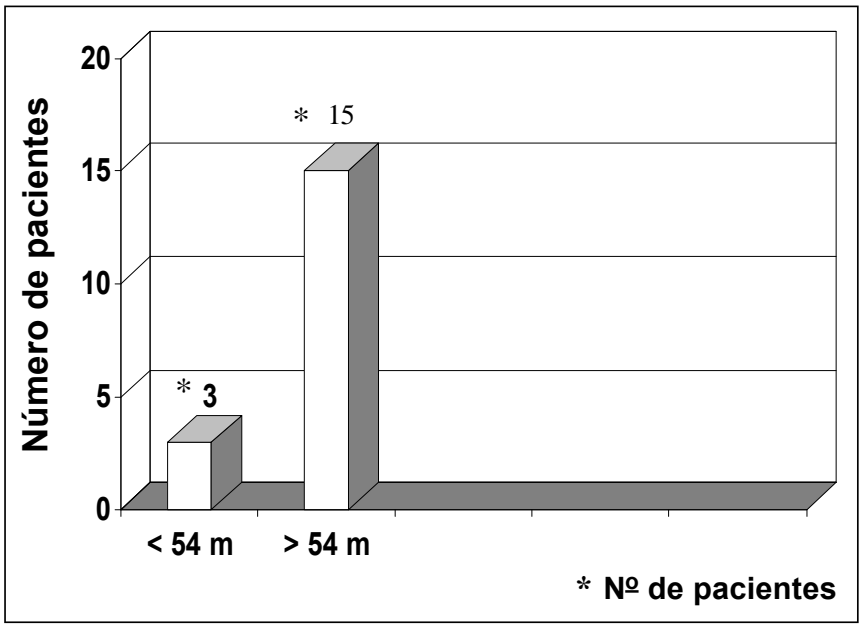

Figura 1 - Aumento da distância percorrida no teste da caminhada de seis minutos, após treinamento

percorrida pós-treinamento apresentou correlação positiva com o VEF$F_{1}(t=2,833 ; p=0,012)$.

0 incremento de carga inferida pelo aumento na velocidade da esteira no final do treinamento não se correlacionou com o incremento na distância percorrida no final do treinamento $(t=0,402 ; p=0,693)$.

Os pacientes apresentaram IMC de $23,2 \mathrm{~kg} / \mathrm{m}^{2}$, em média, DP $=4,1$. Quando se consideraram os valores previstos por faixas etárias constatamos que nove dos 23 pacientes (39\%) apresentaram IMC abaixo do limite inferior. Desses nove pacientes, $6(66 \%)$ atingiram, após treinamento, o mínimo significativo de $54 \mathrm{~m}$ de incremento na distância caminhada e alcançaram o valor previsto para o TC6.

Quando se avaliaram, $\mathrm{f}$ e $\mathrm{SpO}_{2}$ não se observou diferença significativa nos períodos pré e pós-treinamento, no início e no final do TC6. A FC apresentou aumento significativo no final do teste, após treinamento $(p=0,035)$.

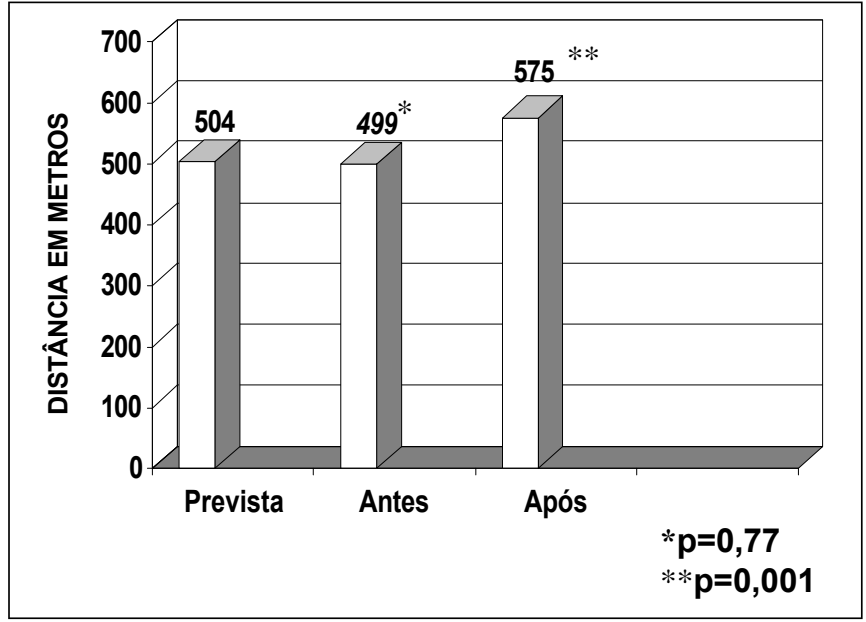

Figura 2 - Médias das distâncias previstas e percorridas no TC6 antes e após treinamento

\section{DISCUSSÃo}

A partir de uma adaptação feita por Cooper (1968), o teste da caminhada passou a ser utilizado na medida do desempenho para exercícios, em programas de reabilitação. Há, todavia, necessidade de padronização na metodologia, especialmente quanto ao tipo e intensidade do incentivo dado ao paciente durante sua realização.

Estudos têm demonstrado o efeito do aprendizado em relação ao teste da caminhada, principalmente se repetidos em curto espaço de tempo ${ }^{(7)}$. Por outro lado, se 0 teste é realizado incentivando-se o paciente, o resultado pode ser melhor ${ }^{(1)}$. Na nossa experiência, o ideal é caminhar ao lado do paciente. 0 incentivo sistemático que utilizamos para os nossos pacientes pode explicar os resultados encontrados pré-treinamento, que não diferem estatisticamente do previsto calculado pela equações de Enright e Sherril, baseado em indivíduos normais e in- 
centivados de maneira diferente, em relação ao nosso protocolo. Os autores utilizaram as seguintes frases de incentivo padronizadas: "Você está indo bem", "Continue fazendo um bom trabalho". Não há relato de acompanhamento do paciente, passo a passo. Em nosso protocolo as frases de incentivo padronizadas buscam sempre melhorar e não manter o desempenho do paciente, que sempre é acompanhado por um membro da equipe.

A capacidade para o exercício melhora após programa de reabilitação pulmonar, quando inferida pela melhora no teste da caminhada ${ }^{(8)}$, e este achado nem sempre se correlaciona com os valores de $\mathrm{VEF}_{1}$ prévios ao treinamento ${ }^{(9)}$. Existem outros fatores que podem influenciar 0 desempenho no teste. Em estudo conduzido por Hamilton et al. ${ }^{(10)}$ observou-se que $70 \%$ dos pacientes com DPOC têm diminuição da musculatura do quadríceps quando comparados com indivíduos normais, com a mesma idade. Além disso, a terapia com corticosteróides pode levar à miopatia, afetando a musculatura respiratória e a esquelética ${ }^{(11,12)}$. A força do quadríceps correlaciona-se com a distância percorrida no TC6 e com a intensidade dos sinto mas apresentados durante o teste incremental de MMII. 0 treinamento da musculatura de mMII melhora o rendimento muscular e 0 teste de endurance de MMII, independente da função pulmonar prévia. Existe correlação entre o consumo máximo de oxigênio ( $\dot{v}_{2}$ máx), a distância percorrida e a taxa de trabalho durante exercício em cicloergômetro ${ }^{(13)}$ e os dados são similares em pessoas normais e naquelas com obstrução ao fluxo aéreo(14). Obtivemos, contudo, correlação positiva entre $O \mathrm{VEF}_{1} \mathrm{e}$ distância percorrida após treinamento, sugerindo tendência a melhores resultados naqueles com menor limitação ao fluxo aéreo. Estudo de Zuwallack et al. (15) sugere que paciente com menor obstrução ao fluxo aéreo tem meIhor potencial para exercício com alta carga de esforço e maior ganho aeróbico após treinamento, durante a reabilitação. Ressaltamos que $87 \%$ dos nossos pacientes atingiram o patamar de melhora considerada significativa, ou seja, $54 \mathrm{~m}$.

Apesar do ganho em distância caminhada, não houve a esperada diminuição da FC no final da caminhada, após treinamento. Todavia, o grau de esforço desenvolvido no pré e no pós-treinamento aos seis minutos de caminhada não são comparáveis, já que houve incremento na distância após treinamento. Assim, parece mais adequado avaliar esse parâmetro após treinamento, no momento em que o paciente atingir a distância caminhada na fase prétreinamento. Por outro lado, a média de $\mathrm{SpO}_{2}$ dos nossos pacientes permaneceu abaixo de $90 \%$ durante o TC6; 0 fato de não se utilizar suplementação de oxigênio, durante 0 teste, pode ter determinado o aumento na FC devido à hipoxemia. A escala de Borg, não utilizada no TC6, nesta série de pacientes, poderia ter auxiliado na avaliação do desempenho. Contudo, o incremento na distância caminhada e na carga de trabalho pressupõe melhora na performance dos pacientes.

Como já está exaustivamente relatado na literatura(1), não há ganho de função pulmonar após programas de reabilitação, o que foi constatado nos nossos dados.

A falta de correlação entre 0 incremento de carga de treinamento, inferida pela velocidade da esteira, e o aumento na distancia percorrida no teste de caminhada pode ser explicada por resposta heterogênea ao exercício. Mesmo aqueles com níveis altos de carga de treinamento podem ter resposta aquém do esperado, por não terem atingido o nível e tempo adequados de treinamento em um protocolo não personalizado. 0 contrário pode ocorrer com aqueles que atingiram baixas cargas de treinamento. Outros fatores podem ter influenciado, como a troficidade da musculatura periférica, idade, limitação funcional e hipoxemia. Neder et al.(16) observaram que pacientes que atingiram o limiar anaeróbio no teste de esforço máximo pré-treinamento não obtiveram incremento aeróbio após treinamento. Relacionaram o maior ganho aeróbio a pacientes mais jovens com menor disfunção respiratória e melhor estado nutricional.

Em nosso estudo, apesar de 39\% dos pacientes apresentarem algum grau de disfunção nutricional inferido pelo IMC abaixo do limite inferior, a maioria destes (66\%) atingiu o TC6 previsto e apresentou incremento significativo, na distância percorrida após treinamento. Todavia, o TC6 previsto foi calculado a partir da equação de Enright e Sherril, cujos resultados, como já referimos, podem ser subestimados. Por outro lado, não utilizamos parâmetros que inferem a massa ou força muscular dos membros inferiores, especialmente do quadríceps, o que poderia levar a resultados diferentes. Há necessidade, também, de um grupo maior de desnutridos, para a efetiva avaliação da interferência no TC6.

Concluindo, sugerimos utilizar metodologia padronizada e adequada para realização do teste da caminhada de seis minutos, inclusive quando o objetivo for obter valores previstos ou referenciais. As equações de Enright e Sherril, obtidas a partir de indivíduos normais, parecem tender a valores subestimados, já que não diferem, estatisticamente, dos valores registrados pré-treinamento em nossos pacientes com DPOC. Outros trabalhos poderão contribuir para confirmar esses achados.

Reafirmamos o ganho de performance física, após reabilitação, independente do estado funcional inicial, o que justifica sua indicação, mesmo para pacientes com limitação respiratória acentuada. Parece, contudo, haver tendência de maior ganho em pacientes com menor disfunção respiratória.

A resposta heterogênea à carga de treinamento em pacientes com DPOC sugere a necessidade de estudar os 
fatores preditivos e determinantes da melhor resposta após reabilitação nesses pacientes. Isso possibilitaria discriminar grupos, cujas peculiaridades exigem ajustes e personalização do treinamento, visando maior ganho possível em performance física.

\section{Agradecimentos}

Os autores agradecem ao Prof. Dr. J osé Roberto J ardim, da Disciplina de Pneumologia (Unifesp-EPM), pelas valiosas sugestões durante a redação do presente trabalho, e ao Prof. Gercino Monteiro Filho, do IME/UFG, pela análise estatística.

\section{REFERÊNCIAS}

1. American Thoracic Society. Pulmonary rehabilitation. Am J Respir Crit Care Med 1999;159:1666-1682.

2. Enright PI, Sherril DI. Reference equations for the six minute walk in healthy adults. Am J Respir Crit Care Med 1998;158:1384-1387.

3. American Thoracic Society. Standardization of spirometry. Am Rev Respir Dis 1987;136:1285-1298.

4. Sociedade Brasileira de Pneumologia e Tisiologia. I consenso brasileiro sobre espirometria. J Pneumol 1996;22:105-164.

5. Dehoog S. Avaliação do estado nutricional. In: Mahan LK, EscottStump S, eds. Krause: alimentos nutrição e dietaterapia. 9a ed. São Paulo: Roca, 1998;381 e 972.

6. Redelmeier DA, Bayroumi AM, Goldstein RS, Guyatt GH. Interpreting small differences in functional status: the six minutes walk test is chronic lung disease patients. Am J Respir Crit Care Med 1997;155: 12781282.

7. MCG avin CR, Gripta SP, MCHardy GJR. Twelve-minute walking test for assessing disability in chronic bronchitis. Br Med J 1976;1:822823.

8. Goldstein RS, Gort EH, Avendano MA, Gruyatt GH. Randomized controlled trial of respiratory rehabilitation. Lancet 1994;344:1394-1397.

9. Casaburi R, Porszasz J, Burns MR, Carithers ER, Chang RSY, Cooper CB. Physiology benefits of exercises training in rehabilitation of patients with severe chronic obstructive pulmonary disease. Am J Respir Crit Care Med 1997;155:1541-1551.

10. Hamilton Al, Killian KJ, Summers E, J ones NL. Muscle strength symptom intensity and exercise capacity in patients with cardiorespiratory disorders. Am J Respir Crit Care Med 1995;152:2021-2031.

11. American Thoracic Society. Skeletal muscle abnormalities in COPD. Am J Respir Crit Care Med 1999;159:S10-18.

12. American Thoracic Society. Effects of interventions on skeletal muscle dysfunction in COPD. Am J Respir Crit Care Med 1999;159:S19-25.

13. Ribeiro AS, J ardim JR, Nery LE. Avaliação da tolerância ao exercício em pacientes com doença pulmonar obstrutiva crônica. Teste da caminhada de seis minutos versus cicloergometria. J Pneumol 1994;20: 112-116.

14. Lewis MI. The relationship between oxygen consumption and work rate in patients with airflow obstruction. Chest 1994;106:366-372.

15. Zuwallack RL, Patel K, Reardon JZ, Clark BA, Normandin EA. Predictors of improvement in the 12-minute walking distance following a sixweek outpatients rehabilitation program. Chest 1991;99:805-808.

16. Neder J A, Nery LE, Cendon SP, Ferreiras IM, J ardim J R. Reabilitação pulmonar: fatores relacionados ao ganho aeróbico de pacientes com DPOC. J Pneumol 1997;23:115-123. 